\title{
Efficient and Robust Multi-Objective Optimization of Food Processing: A Novel Approach with Application to Thermal Sterilization
}

\author{
José Oscar H. Sendín, Antonio A. Alonso, Julio R. Banga* \\ Process Engineering Group, IIM-CSIC \\ Consejo Superior de Investigaciones Científicas \\ C/Eduardo Cabello, 6, 36208 Vigo, Spain
}

\begin{abstract}
The optimisation of thermal processing of foods is a topic which has received great attention during the last decades. The majority of the authors have considered the use of single objective (criteria) for the optimisation. However, the simultaneous optimisation of several objectives is much more realistic and desirable, but the associated non-linear programming problems can be very challenging to solve. Here, we describe an efficient and robust multi-criteria optimisation method which can be successfully applied to large dynamic systems, like those arising from the modelling of thermal processing of foods. Further, their capabilities for better design and operation of these processes will be highlighted with selected case studies, where the generated Pareto solutions will be analysed. Finally, we will also illustrate their advantages over other recently proposed strategies.
\end{abstract}

Keywords: multi-objective optimization, food thermal processing, Pareto solutions

\footnotetext{
${ }^{*}$ Corresponding author. Tel.: +34 986214473. Email address: julio@iim.csic.es (J.R. Banga)
} 


\section{INTRODUCTION}

23

24

25

26

27

28

29

Model-based optimization of food processing is a topic which has received great attention during the last decades, benefiting from all the significant advances already made in computer aided-engineering. Recent works arising from the modelling of thermal processing of foods (like e.g. those of Balsa et al., 2002a, 2002b; Chalabi et al., 1999; Chen and Ramaswamy, 2002; García et al., 2006; Miri et al., 2008; Simpson et al., 2008) deal fundamentally with optimal control problems where the use of a single performance index (objective function or criteria) is considered for the optimization.

When focusing on the thermal sterilization of canned foods, the aim of process optimization often consists of finding the heating temperature profile and the process time maximizing the final nutrient retention of a conduction-heated canned food while assuring a certain microbiological lethality. However, other criteria can be considered, such as minimization of total process time, maximization of energy efficiency, etc. Full reviews of optimization methods and applications in food process engineering, including thermal processing, have been presented by Banga et al. (2003) and Durance (1997).

For many problems of interest the simultaneous optimization of multiple objectives (e.g. product quality, operating costs, capital investment, etc.) is a more realistic and desirable approach, but since these criteria are frequently opposing the optimal solution is not unique. Furthermore, the associated non-linear programming problems (NLPs) can be very challenging to solve. The purpose of multi-objective, or multicriteria, optimization is, ideally, to generate the set of solutions involving optimal trade-offs among the different objectives, i.e. the set of solutions which represent the relatively best alternatives. In this regard, the common optimization problem for nutrient retention can be in fact considered as a multi-objective optimization problem, since there exist two conflicting criteria: the system should remain for a certain period of time at a high enough temperature in order to achieve the desired minimum lethality. On the other hand, nutrients are destroyed by the action of heat. A compromise between both criteria should be found, although it is generally accepted 
51 that microbiological safety must be the primary objective and, consequently, canned foods are usually over-processed (Fryer and Robbins, 2005).

In the field of food engineering, multi-criteria optimization has received little attention. Some authors have studied the optimization of thermal processing of canned foods using several objective functions (Banga et al., 1991; Noronha et al., 1996; Erdogdu and Balaban, 2003), but very little attempts have been made to solve the complete multi-objective optimization problem (i.e. to find a representative set of solutions capturing the complete trade-off among the different criteria). Kiranoudis and Markatos (2000) considered the multi-objective design of food dryers using a static mathematical model. These authors minimize simultaneously an economic measure and the colour deviation of the final product. Olmos et al. (2002) studied the compromise between the final product quality and process time by using the so-called $\varepsilon$-constraint approach, i.e. the final product quality is maximized repeatedly subject to a constraint on the allowed total drying time, which is varied in each optimization. However, no work has been found discussing the possible difficulties and pitfalls which often appear regarding the multi-objective optimization of nonlinear dynamic food processing models.

In this contribution, which is an extension of the work originally presented by Sendín et al. (2004), we describe a novel multi-criteria optimisation method which can be successfully applied to large and complex dynamic systems. The efficiency and robustness of this approach is illustrated with case studies.

\section{MULTI-OBJECTIVE OPTIMIZATION OF DYNAMIC SYSTEMS}

The aim of a general multi-objective (or multi-criteria) optimization of a dynamic system is to find the $n_{v}$ static design variables $\boldsymbol{V}$ and the $n_{u}$ time-dependent manipulated variables or controls $\boldsymbol{u}(t)$ which minimize (or maximize) simultaneously a vector of $m$ performance indexes of the system $\left(J_{m}\right)$ subject to a number of constraints. Mathematically, this general non-linear, constrained, multi-objective optimization problem (MOP) is stated as follows: 


$$
\min \mathbf{J}=\left[\begin{array}{c}
J_{1}\left(\boldsymbol{y}, \boldsymbol{y}_{t}, \boldsymbol{y}_{\xi}, \boldsymbol{y}_{\xi \xi}, \boldsymbol{z}, \dot{\boldsymbol{z}}, \boldsymbol{u}, \boldsymbol{v}, \xi, t\right) \\
J_{2}\left(\boldsymbol{y}, \boldsymbol{y}_{t}, \boldsymbol{y}_{\xi}, \boldsymbol{y}_{\xi \xi}, \boldsymbol{z}, \dot{\boldsymbol{z}}, \boldsymbol{u}, \boldsymbol{v}, \xi, t\right) \\
\vdots \\
J_{m}\left(\boldsymbol{y}, \boldsymbol{y}_{t}, \boldsymbol{y}_{\xi}, \boldsymbol{y}_{\xi \xi}, \boldsymbol{z}, \dot{\boldsymbol{z}}, \boldsymbol{u}, \boldsymbol{v}, \xi, t\right)
\end{array}\right]
$$

80

81

82

83

84

85

86

87

Subject to:

$$
\begin{aligned}
& \boldsymbol{\Psi}\left(\boldsymbol{y}, \boldsymbol{y}_{t}, \boldsymbol{y}_{\xi}, \boldsymbol{y}_{\xi \xi}, \boldsymbol{z}, \dot{\boldsymbol{z}}, \boldsymbol{u}, \boldsymbol{v}, \xi, t\right)=\mathbf{0} \\
& \boldsymbol{\Psi}_{\mathbf{0}}\left(\boldsymbol{y}, \boldsymbol{y}_{t}, \boldsymbol{y}_{\xi}, \boldsymbol{y}_{\xi \xi}, \boldsymbol{z}, \dot{\boldsymbol{z}}, \boldsymbol{u}, \boldsymbol{v}, \xi, t_{0}=0\right)=\mathbf{0} \\
& \mathcal{B}(y, u, v, \Omega, t)=\mathbf{0} \\
& \boldsymbol{g}\left(\boldsymbol{y}, \boldsymbol{y}_{t}, \boldsymbol{y}_{\xi}, \boldsymbol{y}_{\xi \xi}, \boldsymbol{z}, \dot{\boldsymbol{z}}, \boldsymbol{u}, \boldsymbol{v}, \xi, t\right) \leq \mathbf{0} \\
& \boldsymbol{u}^{L} \leq \boldsymbol{u}(t) \leq \boldsymbol{u}^{U} \\
& \boldsymbol{v}^{L} \leq \boldsymbol{v} \leq \boldsymbol{v}^{U}
\end{aligned}
$$

where Equations (2)-(4) are equality constraints representing the system dynamics, described in general form by a set of partial differential algebraic equations (PDAEs) with appropriate initial and boundary conditions; $\xi \in \Omega \subset \mathbb{R}^{3}$ are the spatial variables; $\boldsymbol{y}(\xi, t) \in \mathbb{R}^{p}$ is the subset of state variables depending on both time and spatial location; $\boldsymbol{z}(t) \in \mathbb{R}^{s}$ is the subset of time-dependent state variables; $\boldsymbol{y}_{t}=\delta \boldsymbol{y} / \delta t ; \boldsymbol{y}_{\xi}$ and $\boldsymbol{y}_{\xi \xi}$ are the first and second order spatial derivatives, respectively; $\dot{\boldsymbol{z}}=d \boldsymbol{z} / d t$; $\boldsymbol{u}(t) \in \mathbb{R}^{n u}$ corresponds to the manipulated time-dependent decision variables, and $v \in \mathbb{R}^{n v}$ are the static decision variables. Eq. (5) includes additional requirements in the form of inequality path and/or final time constraints, and equations (6) and (7) represent upper and lower bounds on the decision variables (superscript $U$ and $L$, respectively). These constraints represent the feasible space $S$, and the set of all possible values of the objectives satisfying the constraints constitutes the objective space $\boldsymbol{O}$. For the sake of clarity, hereafter we will denote by $\boldsymbol{x}$ the vector of decision variables including time-independent variables and controls.

As in the single-objective case, the multi-objective dynamic optimization problem described above can be transformed into a nonlinear multi-objective optimization 
103

problem using e.g. control vector parametrization, CVP (Vassiliadis et al., 1994a,b), and solving the system dynamics as an inner initial value problem, IVP. Partial differential equations can be converted into sets of ordinary differential equations (ODEs) by means of suitable discretization methods (Schiesser, 1991).

Very often the components of the objective vector J are in conflict with each other. In this case, the solution minimizing one of the criteria does not minimize the other, that is, it will not be possible to find a unique solution which is simultaneously optimal for all the objectives (it is clear that if there exists such a point, it will provide a solution to the MOP). Unlike single objective optimization, in a MOP there will be in general multiple points which are optimal in the sense that an improvement in one objective function can only be achieved with a worsening in one or more of the others.

For two or more objectives, the concept of domination is introduced to determine which solutions are better than others. Given two points $\boldsymbol{x}_{1}$ and $\boldsymbol{x}_{2}$, the vector $\boldsymbol{J}\left(\boldsymbol{x}_{1}\right)$ is said to dominate $\boldsymbol{J}\left(\boldsymbol{x}_{2}\right)$ if $J_{i}\left(\boldsymbol{x}_{1}\right) \leq J_{i}\left(\boldsymbol{x}_{2}\right)$ for all $i=1, \ldots, m$, with at least one strict inequality.

A feasible solution $\boldsymbol{x}^{*}$ is said to be local Pareto-optimal (or efficient) if there is no another feasible solution $\boldsymbol{x}$ in the neighborhood of $\boldsymbol{x}^{*}$ such as $\boldsymbol{J}(\boldsymbol{x})$ dominates $\boldsymbol{J}\left(\boldsymbol{x}^{*}\right)$. A feasible solution $\boldsymbol{x}^{*}$ is said to be global Pareto-optimal if there is no another solution $\boldsymbol{x}$ over the entire feasible space such as $\boldsymbol{J}(\boldsymbol{x})$ dominates $\boldsymbol{J}\left(\boldsymbol{x}^{*}\right)$.

A related concept is that of weak Pareto optimality. A solution $\mathbf{x}^{*}$ is weakly Paretooptimal if there does not exist another solution $\boldsymbol{x}$ such that $J_{i}(\boldsymbol{x})<J_{i}\left(\boldsymbol{x}^{*}\right)$, for all $i=1, \ldots, m$.

The set of all Pareto-optimal solutions is usually referred as the Pareto front. In the absence of any further information about the problem, all Pareto-optimal solutions are mathematically equivalent, that is, no solution can be said to be better than another.

When solving a MOP, other points of interest are the utopia vector and the nadir (or pseudo-nadir) vector: 
- The utopia vector $\mathbf{J}^{*}$ is the vector of objective functions containing the individual global minima of the objectives.

- The nadir vector $\mathbf{J}^{\text {Nadir }}$ is the vector of objective functions containing the upper bounds of the objectives in the Pareto-optimal set. The $i^{\text {th }}$ component of the nadir vector can be estimated as $J_{i}^{\text {Nadir }}=\max \left\{J_{i}\left(\boldsymbol{x}_{j}^{*}\right)\right\}$ where $\boldsymbol{x}_{j}^{*}, j=1, \ldots, m$, is the global minimizer of the $j^{\text {th }}$ objective.

\section{MULTI-OBJECTIVE SOLUTION STRATEGY}

A wide range of approaches have been proposed in the last decades to solve MOPs. Reviews of these methods can be found in the books by Miettinen (1999) and Deb (2001), and the references cited therein.

Classical approaches deal with MOPs by means of scalarization techniques, which transform the original MOP into a single-objective non-linear programming (NLP) problem making use of some characteristic parameter, e.g. a vector of weights. Such parameter can either represent the relative importance of the objectives or be a mere mathematical device which is varied systematically to hopefully obtain different solutions.

We want to stress the fact that, in a mathematical sense, all Pareto-optimal solutions (potentially an infinite number) are equivalent and it can not be said that one solution is better than another. Multi-objective optimization implies a decision-making process concerning a large number of optimal alternatives. From a practical point of view, the user is only interested in one final solution. The decision maker (DM) is the responsible for selecting such a solution, who will assign preferences to the objectives using some additional information which quite often is subjective and/or difficult to express in mathematical terms.

In this work we focus on methods that are able to produce a set of Pareto-optimal solutions capturing the complete trade-off among the objectives. This is a crucial aspect for the DM to choose a suitable compromise which will represent more accurately his/her preferences. 
Scalarization techniques require solving repeatedly a set of single NLPs, but very often

it is not obvious how to change the method parameters in order to obtain a satisfactory solution or a good distribution of points. Computing the Pareto-optimal set can be a very challenging task due to the highly constrained and non-linear nature of food processing systems. In this regard, it is important to keep in mind that the majority of the existing implementations ultimately rely on local, gradient-based, optimization routines (e.g. SQP) for solving the NLPs, so they can fail if the MOP is nonconvex; the solution can only be guaranteed to be local Pareto-optimal. This drawback can be addressed by using suitable global optimization (GO) methods (Sendín et. al, 167 2006).

A different approach to solve MOPs is based on the use of evolutionary algorithms, in which there has been an increasing interest in the last decades (Coello, 1999). These methods mimic the mechanisms of natural selection and genetics by using a population of possible solutions. Thus, they can find simultaneously multiple nondominated points in one single optimization run. This fact, together with the ability of these multi-objective evolutionary algorithms (MOEAs) to deal with problems involving non-convex Pareto fronts, makes them attractive to solve highly nonlinear MOP. It should be noted that single-objective evolutionary algorithms can also be used in combination with scalarization techniques.

\section{Methodology: NBI-based Weighted Tchebycheff Approach}

178 In the following paragraphs, we present a novel solution strategy which combines two 179 well known scalarization techniques, the weighted Tchebycheff method and the Normal Boundary Intersection (NBI) method of Das \& Dennis (1998).

\section{Weighted Tchebycheff Method}

The weighted Tchebycheff method, originally introduced in Bowman (1976), is an extension of the traditional min-max formulation, and can be stated as follows:

$$
\min \mathrm{U}_{\mathrm{F}}=\max \left\{w_{i}\left|J_{i}(\boldsymbol{x})-J_{i}^{0}\right|\right\}
$$
taken as reference point, minimizing function $U$ can provide a complete representation 
of the Pareto-optimal set with a variation in the weights. Additionally, this formulation assures that the solution is at least weak Pareto optimal (Koski and Silvennoinen, 1987; Miettinen, 1999). Once more, the difficulty of this approach lies in the determination of the parameters to ensure a good representation of the optimal front by solving as few NLPs as possible.

192

193

\section{Normal Boundary Intersection (NBI)}

The original NBI method essentially works by solving a set of NLPs of the form:

$$
\max U_{F}=\lambda
$$

subject to:

$$
\Phi \mathbf{w}+\lambda \vec{n}=\mathbf{J}(\boldsymbol{x})-\mathbf{J}^{*}
$$

$\Phi$ is a $m \times m$ pay-off matrix in which the ith column is $\mathbf{J}\left(\boldsymbol{x}_{i}^{*}\right)-\mathbf{J}^{*}$ (i.e. the objective functions are shifted to the origin); $\mathbf{w}$ is a vector of weights such that $\left(\sum_{i=1}^{m} w_{i}=1\right)$ and $w_{i} \geq 0 ; \vec{n}=\Phi \vec{e}$ is the unitary quasi-normal vector, being $\vec{e}$ a vector of ones. The product $\Phi \mathbf{w}$ defines a point in the so-called Convex Hull of Individual Minima (CHIM). The intersection between the normal to the CHIM starting from this point and the boundary of the objective space closest to the origin is expected to be Pareto-optimal (Figure 1a).

The above NLP (called NBI-subproblem) is solved for various weight vectors $\mathbf{w}$ in such a way that an equally distributed set of them produces an even spread of solutions in the boundary of the objective space. It should be noted that the equality constraints introduced by NBI assure that the solution is actually on the normal to the CHIM.

\section{Hybrid Approach}

In this work we have combined both methods described previously in order to surmount their respective drawbacks, and more specifically, those concerning with the NBI programming technique:

1) Generation of local optimal solutions with non-convex Pareto fronts, which makes necessary the use of suitable global optimization solvers. 
2) The NBI method can also yield non Pareto optimal points if the normal to the CHIM intersects the objective space boundary in a non-convex region. Although non-optimal solutions can be eliminated after computing the Pareto front by means of e.g. a "Pareto filter", it is clear that solving an NLP whose solution does not belong to the Pareto optimal set is a computational waste.

3) The NBI method introduces an additional variable and additional equality constraints (as many as objective functions), which can increase the computational cost of computing the Pareto front. This issue is especially important when using global stochastic optimization methods to solve the associated NLPs, since this class of algorithms are not usually designed to handle equality constraints. Also, reasonable bounds for the method variable $\lambda$ must be fixed in order to help the search process.

Recent attempts to improve NBI with different reformulations can be found in e.g. Lim et al. (2001), Messac \& Mattson (2004) and Shukla (2007).

Our strategy makes use of the NBI programming technique to generate a series of reference points on the hypercube defined by the global minimizers of the objective functions. These points are used to solve a weighted min-max problem, using weights which are determined from the quasi-normal direction to the CHIM (Figure 1b).

Thus, for a given weight vector $\boldsymbol{w}_{k}$, which defines the point $P_{k}=\Phi \boldsymbol{w}_{k}$ on the CHIM, the $k$ th NLP to solve is formulated as:

$$
\min \mathrm{U}_{\mathrm{F}}=\max \left\{\bar{w}_{k, i}\left(J_{i}(\boldsymbol{x})-J_{k, i}^{0}\right)\right\}
$$

where, omitting the mathematical derivation:

$$
\begin{aligned}
& \boldsymbol{J}_{k}^{0}=\boldsymbol{J}^{*}+\left(\Phi \boldsymbol{w}_{k}+\bar{\lambda}_{m} \vec{n}\right) \\
& \bar{w}_{k, i}=-\frac{1 / \vec{n}_{i}}{\sum_{i=1}^{m} 1 / \vec{n}_{i}}
\end{aligned}
$$

and $\bar{\lambda}_{m}$ is determined by solving a linear programming (LP) problem defined as finding $\bar{\lambda}_{m}$ and the 'dummy' vector $\overline{\boldsymbol{J}}_{d}$ to: 


$$
\max \mathrm{U}_{\mathrm{F}}{ }^{\prime}=\bar{\lambda}
$$

subject to:

242

$$
\mathbf{J}^{*} \leq \bar{J}_{d} \leq \mathbf{J}^{\text {Nadir }}
$$

\section{Multi-Objective Optimization Process}

The proposed multi-objective optimization process is composed of the following steps:

1) Search for the global individual optima of the objectives, which will provide a first insight into the trade-off involved among the criteria.

2) Generate a set of Kequally spaced weight vectors.

3) For each weight vector $\boldsymbol{w}_{k}, k=1, \ldots K$ :

a) Find the reference point $\mathbf{J}_{k}^{0}$ (equation 12 ) and the weight vector $\overline{\boldsymbol{w}}_{k}$ (equation 13)

b) Solve the associated NLP (equation 11 ) by means of a suitable singleobjective optimization method.

4) Analysis of solutions and selection.

The strategy described above ultimately requires the solution of a set of singleobjective NLPs, so the use of robust and efficient GO methods becomes a critical issue. Thus, we have implemented the hybrid NBI-Tchebycheff approach coupled with different GO solvers in order to provide a greater flexibility to select the most appropriate method for the problem under consideration.

It is worth mentioning that, since the associated NLPs are solved sequentially, the solutions of the problems that have been already found can be used in the next ones as initial guesses for the optimization, increasing thus the efficiency of the GO solver. 


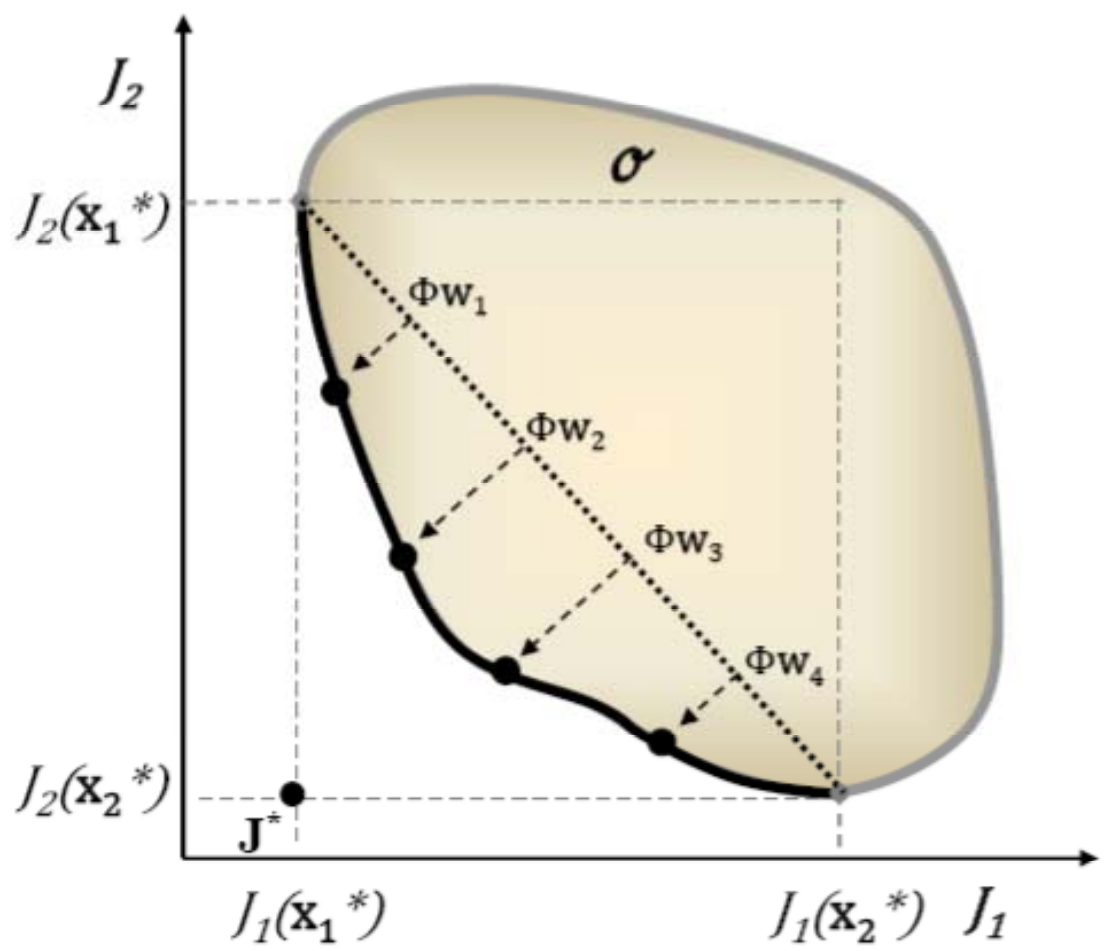

Figure 1a. How NBI works.

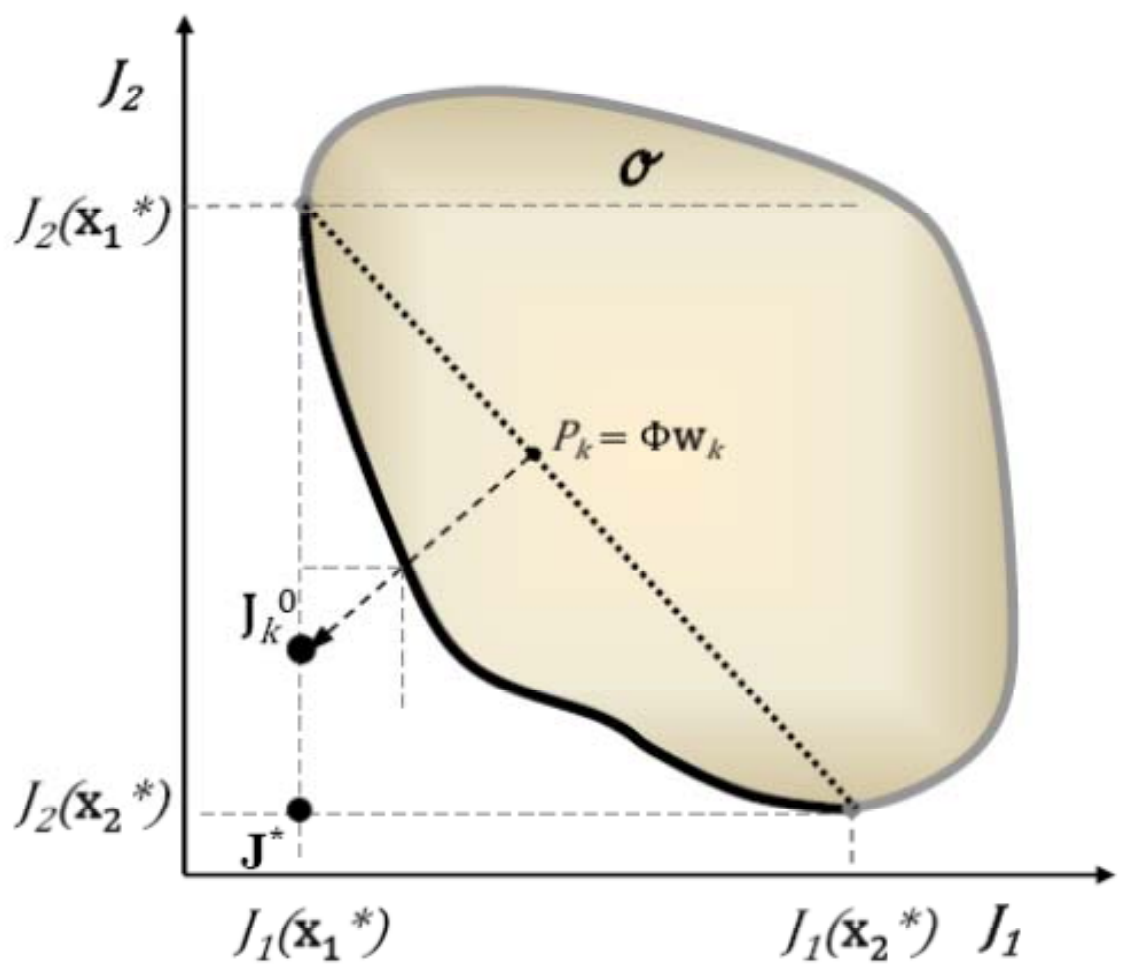


268

269

270

271

272

273

274

275

276

277

278

279

280

281

282

283

284

285

286

287

288

289

290

When studying this class of problems, the aim of the optimization task has been traditionally to find the retort temperature, $\operatorname{Tret}(t)$, which maximizes the final retention of a nutrient or a quality factor (Banga et al., 1991; Noronha et al., 1996). In this contribution, we will consider several case studies which imply the simultaneous optimization of several criteria.

Assuming a finite, isotropic, homogeneous cylinder of radius $R$ and half-height $L$ filled with conduction-heated canned food with a thermal diffusivity coefficient $\alpha$, the heat transfer dynamics is given by (Fourier equation):

$$
\frac{\partial T}{\partial t}=\alpha\left(\frac{\partial^{2} T}{\partial r^{2}}+\frac{1}{r} \frac{\partial T}{\partial r}+\frac{\partial^{2} T}{\partial z^{2}}\right)
$$

with the following boundary and initial conditions:

$$
\begin{aligned}
& T(R, z, t)=T_{\text {ret }}(t) \\
& T(r, L, t)=T_{\text {ret }}(t) \\
& \frac{\partial T}{\partial r}(0, z, t)=0 \\
& \frac{\partial T}{\partial z}(r, 0, t)=0 \\
& T(r, z, 0)=T_{0}
\end{aligned}
$$

The numerical method of lines (NMOL) was used to solve the system of PDEs describing the model, and LSODE was employed as the IVP solver for the resulting ODE system.

\section{Case Study A: Constant Retort Temperature (CRT) Process}

This illustrative case study is concerned with the thermal sterilization of canned tuna fish. We consider here the usual CRT process, i.e. a batch thermal process of constant heating temperature followed by a constant cooling stage. Since no specific sterilizer is defined, the come-up and cool-down times are neglected. 
291 For a given system of volume $V_{T}$ and initial temperature $T_{0}$, the aim of the multi292 criteria optimization problems is to find the constant heating temperature $\left(T_{\text {ret }}\right)$ so 293 that after a certain process time $\left(t_{f}\right)$ two objective functions are maximized:

294 1. Overall retention of a nutrient, thiamine:

$$
C_{T h}=\frac{1}{V_{T}} \int_{0}^{V_{T}} \exp \left(\frac{-\ln 10}{D_{C, \text { ref }}} \int_{0}^{t_{f}} \exp \left(\frac{T(r, z, t)-T_{C, r e f}}{Z_{C, \text { ref }}} \ln 10\right) d t\right) d V
$$

2. Surface retention of a quality factor, luminosity:

$$
L_{H}=\exp \left(\frac{-\ln 10}{D_{Q, \text { ref }}} \int_{0}^{t_{f}} \exp \left(\frac{T(t)-T_{Q, \text { ref }}}{Z_{Q, \text { ref }}} \ln 10\right) d t\right)
$$

Imposing the following set of constraints:

299

300

301

302

303

304

305

306

307

308

309

310

311

312 The problem defined above is solved for two different cans (RO-100 and RO-1150).

313 Kinetics for the thermal degradation of the nutrient, the quality factor and the 314

- The heat transfer equation for conduction (eq. 17) with boundary and initial conditions specified by equations (18-22).

- A constraint on the final temperature in the hottest point:

$$
T\left(r, z, t_{f}\right) \leq T_{0}
$$

- A constraint on the final lethality at final time:

$$
F_{S}\left(t_{f}\right) \geq F_{S, D}
$$

with

$$
F_{S}\left(t_{f}\right)=D_{M, r e f} \log \left(\frac{1}{V_{T}} \int_{0}^{V_{T}} \exp \left(\frac{-\ln 10}{D_{M, r e f}} \int_{0}^{t_{f}} \exp \left(\frac{T(r, z, t)-T_{M, r e f}}{Z_{M, r e f}} \ln 10\right) d t\right) d V\right)
$$

where $D_{C, \text { ref }}, D_{Q, \text { ref }}$ and $D_{M, r e f}$ are the time required to reduce the concentration of the nutrient, quality factor and microorganisms, respectively, by a factor of 10 at a certain temperature, $T_{\text {ref }}$; and $Z_{C, \text { ref }}, Z_{Q, \text { ref }}$ and $Z_{M, \text { ref }}$ represent the temperature increase necessary for reducing $D_{C, r e f}, D_{Q, \text { ref }}$ and $D_{M, \text { ref }}$, respectively, by a factor of 10. microorganism are taken from Banga et al. (1993), and are collected in Table 1. 


\section{Case Study B: Variable Retort Temperature (VRT) Process}

316 The second example deals with computing optimal operating policies in processes in 317 which the retort temperature is modulated during the sterilization. The aim of the 318 multi-criteria optimization problem is to find the time-dependent temperature (the 319 control) $T_{\text {ret }}(t)$ over a time horizon to simultaneously:

320 1. Maximize the overall retention of a nutrient, $R_{C G}$ (as in Eq. 23).

3212 2. Maximize the surface retention of a quality factor, $R_{Q S}$ (as in Eq. 24).

322 3. Minimize the total process time, $t_{f}$.

323 These three objectives are subject to the same set of constraints defined in the 324 previous case. Parameters and kinetics (Table 1) are taken from Banga et al. (1991) and 325 Teixeira et al. (1975).

326 The superficial retention for the quality factor has been computed using kinetic data 327 for thiamine. Although such retention has no direct physical significance, these 328 parameters are similar to those associated with the foodstuff surface, e.g. browning 329 reactions and loss of luminosity (Ohlsson, 1980). 
331 Table 1.

332 Parameters for the thermal sterilization case studies

\begin{tabular}{|c|c|c|c|}
\hline & \multicolumn{2}{|c|}{ Case Study A } & Case Study B \\
\hline Product & \multicolumn{2}{|c|}{ Tuna fish } & Pork puree \\
\hline Can & RO-100 & RO-1150 & $307 \times 409$ \\
\hline Diameter (m) & 0.0652 & 0.15 & 0.0875 \\
\hline Height (m) & 0.03 & 0.07 & 0.1160 \\
\hline$\alpha\left(\mathrm{m}^{2} \mathrm{~s}^{-1}\right)$ & \multicolumn{2}{|c|}{$1.143 \cdot 10^{-7}$} & $1.5443 \cdot 10^{-7}$ \\
\hline$T_{O}(\stackrel{\circ}{ } \mathrm{C})$ & \multicolumn{2}{|c|}{25.0} & 71.11 \\
\hline Microorganism & \multicolumn{2}{|c|}{ Clostridium botulinum } & $\begin{array}{c}\text { Bacillus } \\
\text { stearothermophillus }\end{array}$ \\
\hline$Z_{M, r e f}(\stackrel{\circ}{ } \mathrm{C})$ & \multicolumn{2}{|c|}{10.0} & 10.0 \\
\hline$D_{M, r e f}(\mathrm{~s})$ & \multicolumn{2}{|c|}{15.0} & 240.0 \\
\hline Lethality (min) & \multicolumn{2}{|c|}{$\mathrm{F}_{\mathrm{S}} \geq 9.0$} & $\mathrm{~F}_{\mathrm{S}} \geq 20.0$ \\
\hline Nutrient & \multicolumn{2}{|c|}{ Thiamine } & Thiamine \\
\hline$Z_{C, \text { ref }}(\stackrel{\circ}{ } \mathrm{C})$ & \multicolumn{2}{|c|}{31.4} & 25.56 \\
\hline$D_{C, r e f}(\mathrm{~s})$ & \multicolumn{2}{|c|}{5430.0} & 10716.0 \\
\hline$T_{C, \text { ref }}(\stackrel{\circ}{ } \mathrm{C})$ & \multicolumn{2}{|c|}{121.11} & 121.11 \\
\hline Quality Factor & \multicolumn{2}{|c|}{$\begin{array}{c}\text { Luminosity } \\
\text { (L-Hunter value) }\end{array}$} & Thiamine* \\
\hline$Z_{Q, \text { ref }}(\stackrel{\circ}{ } \mathrm{C})$ & \multicolumn{2}{|c|}{44.0} & 25.56 \\
\hline$D_{Q, r e f}(\mathrm{~s})$ & \multicolumn{2}{|c|}{88000.0} & 10716.0 \\
\hline$T_{Q, \text { ref }}(\stackrel{\circ}{ } \mathrm{C})$ & \multicolumn{2}{|c|}{121.11} & 121.11 \\
\hline
\end{tabular}




\section{RESULTS AND DISCUSSION}

\section{Case Study A: CRT Process}

335 Although this problem is somewhat trivial to solve, it is included here for the sake of

336 illustration. Pareto fronts for both RO-100 and RO-1150 cans are depicted in Figure 2.

337 From an inspection of both figures, it can be seen that multi-objective optimization

338 produces Pareto curves with a practically inexistent trade-off, which depends slightly

339 on the dimensions of the can. For the bigger one (RO-1150), the variation in the

340 retention of thiamine and luminosity are meaningless (approximately one percent

341 unit), but the optimum retort temperatures increases along the Pareto-optimal set

342 from $115 \stackrel{\circ}{\circ} \mathrm{C}$ (maximum $L_{H}$ ) to $121{ }^{\circ} \mathrm{C}$ (maximum $C_{T h}$ ). These values are in fact rather

343 similar to those used in industrial practice.

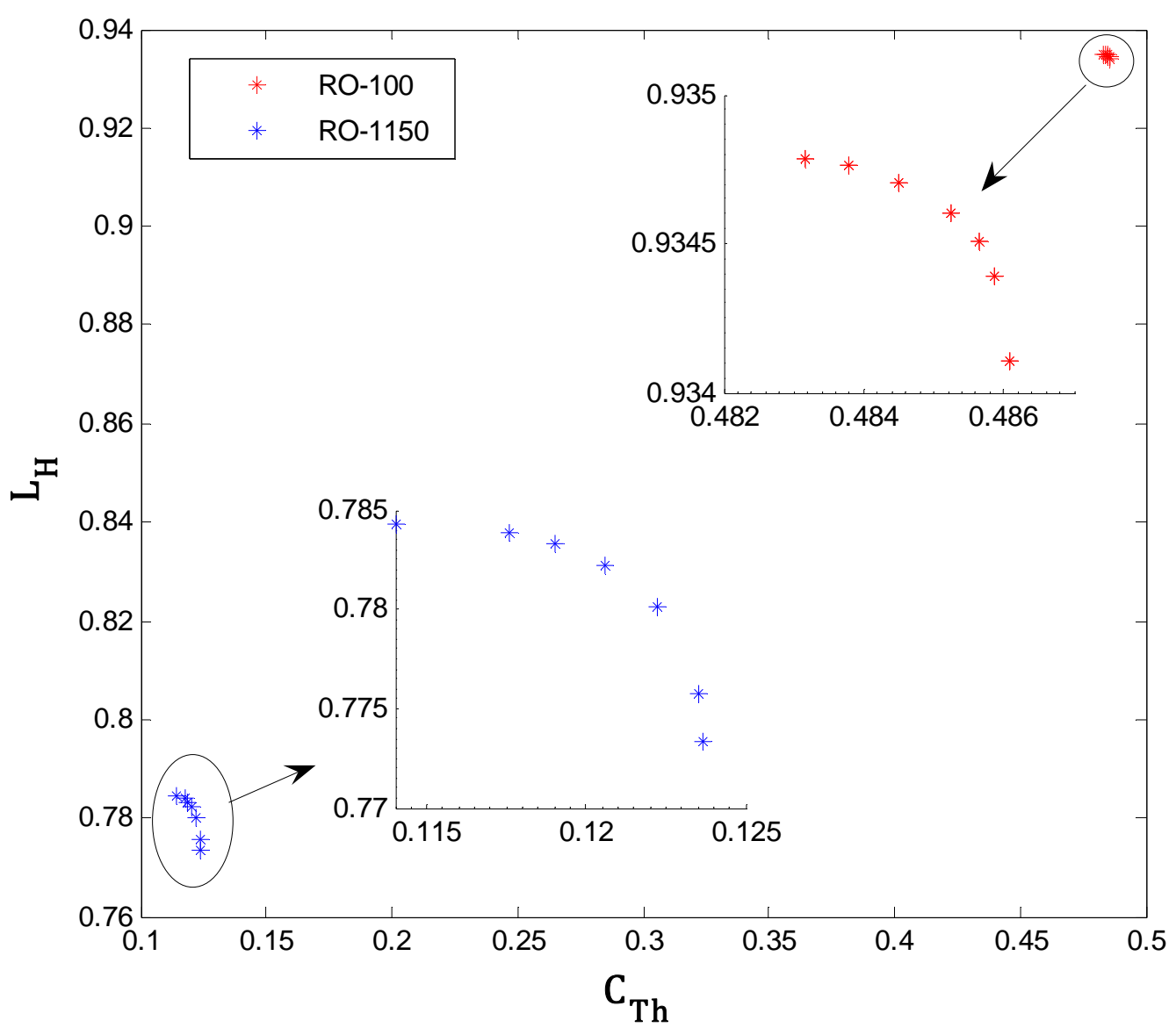

344

Figure 2. Pareto-optimal sets obtained for Case Study A 
346 In Figure 3, the values of the overall and surface retentions versus the optimal 347 constant retort temperatures corresponding to the Pareto set are depicted.
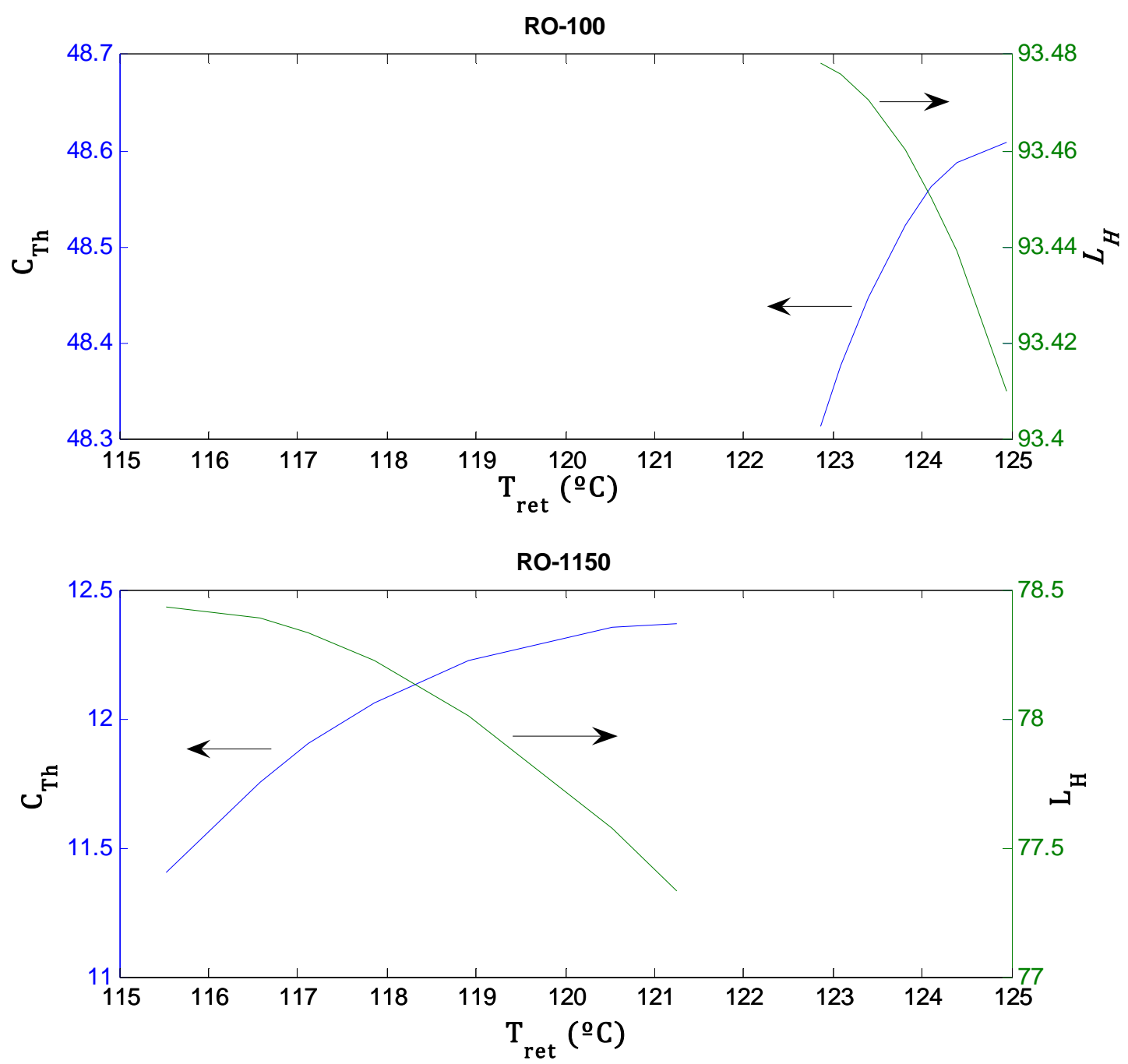

348

Figure 3. Overall and surface retentions vs. constant retort temperatures for Case Study A.

\section{Case Study B: VRT Process}

352 As mentioned previously, suitable single-objective GO methods are needed in order to

353 find global optimal solutions. It is worth mentioning that this problem causes the

354 failure of most SQP-based solvers due to the noise introduced by the numerical 355 integration of the PDEs, which produces a non-smooth objective function (results not 356 shown). 
357 After a preliminary screening of several GO solvers, we have selected the stochastic 358 algorithm Differential Evolution (DE) of Storn \& Price (1997) for the optimization of the 359 individual objectives and the NLPs associated to the multi-objective programming 360 technique. Optimization settings for all the NLPs are as follows:

- Population size: 100 individuals.

- Maximum number of iterations: 300.

- Other solver parameters: default values.

364 The Pareto-optimal set obtained with the hybrid NBI-weighted Tchebycheff approach 365 coupled with DE is plotted in Figure 4, and projections of the retention of both nutrient and quality factor versus process time are depicted in Figures 5 and 6 , respectively.

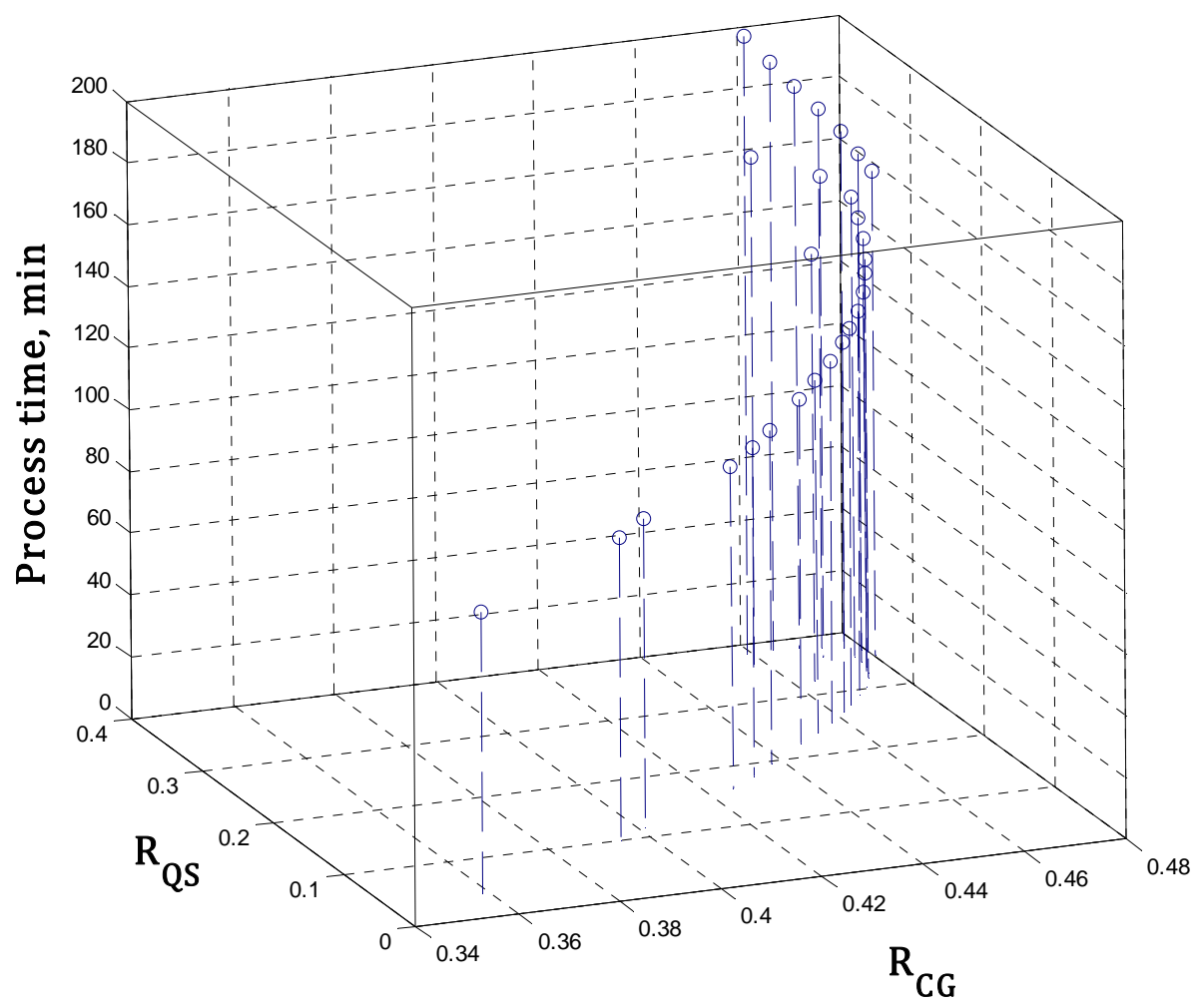

367

Figure 4. Pareto-optimal set obtained for Case Study B using the hybrid NBI-Weighted Tchebycheff approach 


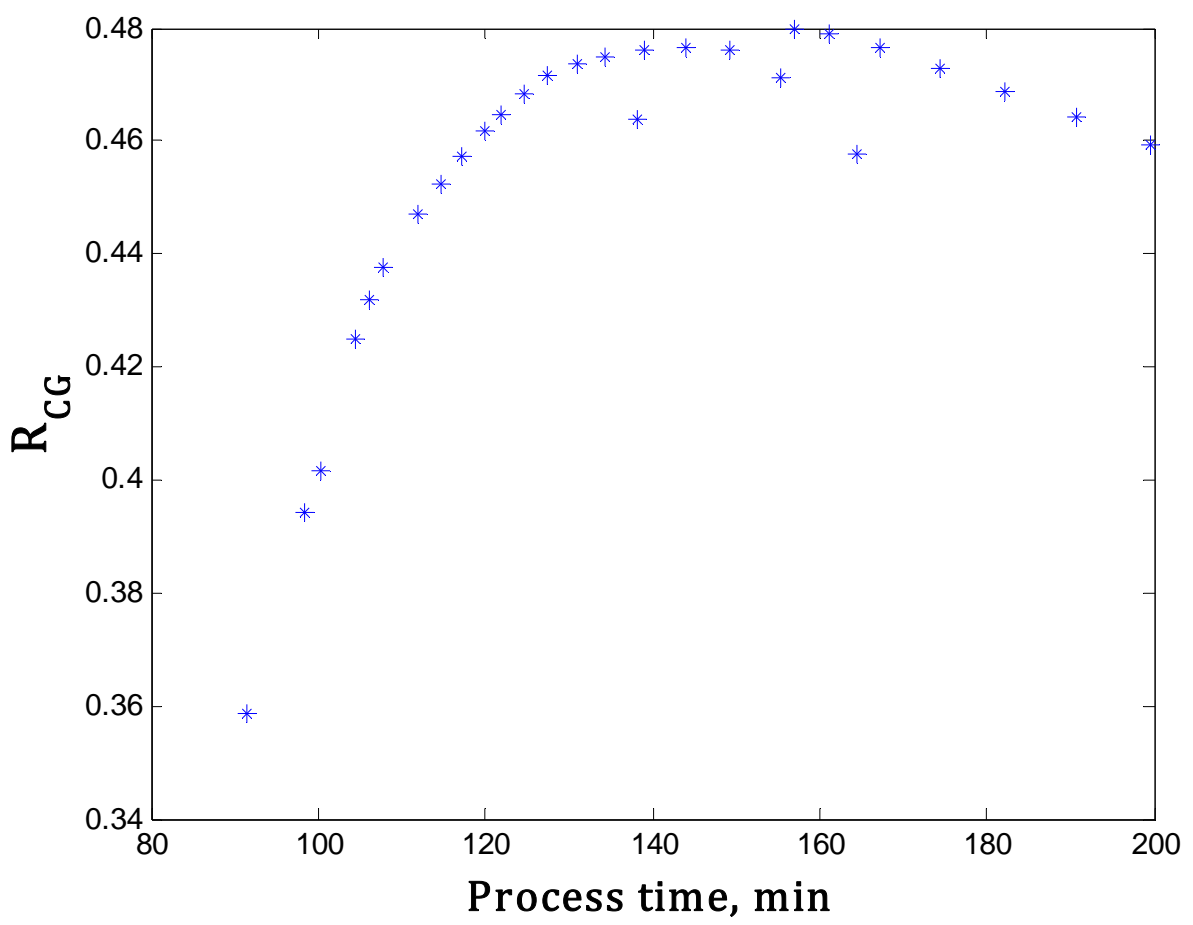

371 Figure 5. Nutrient retention versus process time for the Pareto optimal solutions

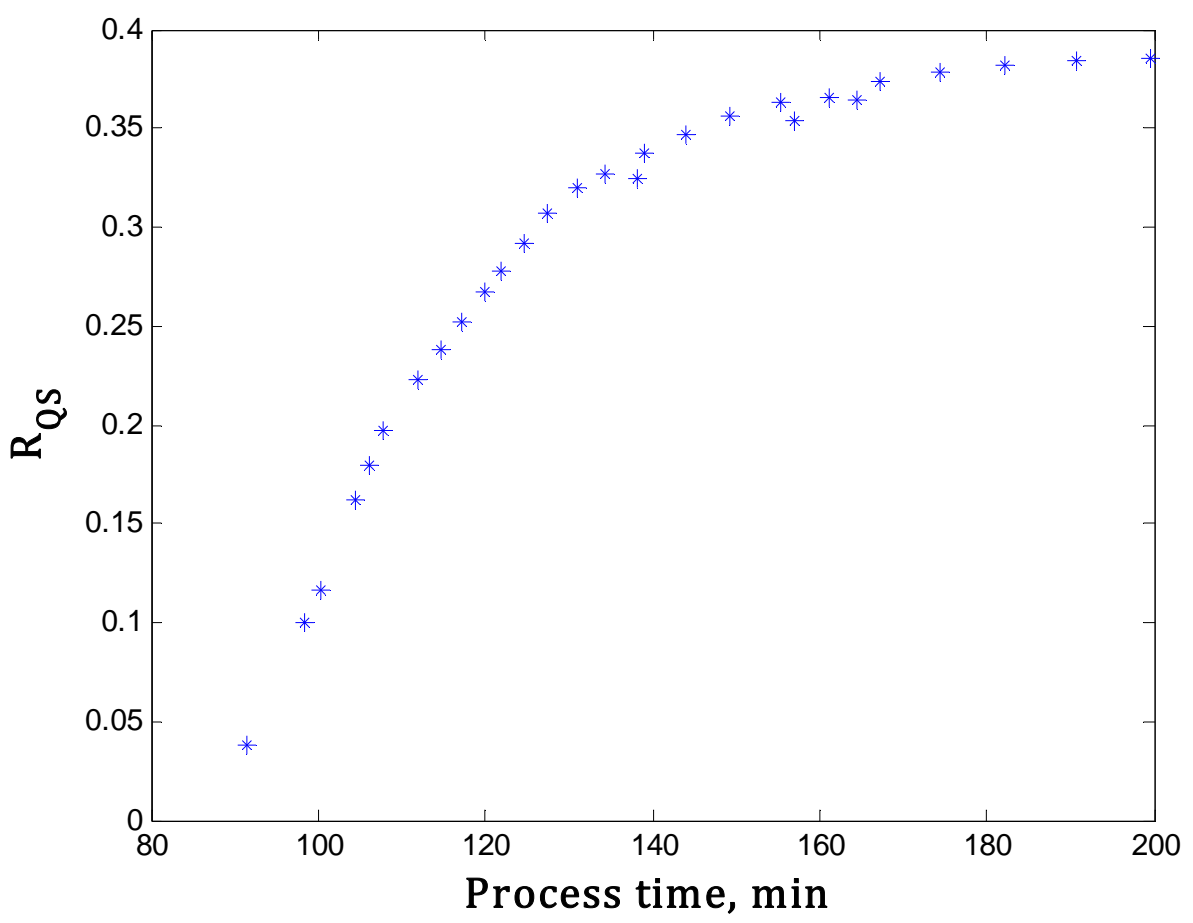

372

373 Figure 6. Superficial retention versus process time for the Pareto optimal solutions 
374 These illustrations show several conflicting scenarios, which are summarized in the 375 pay-off matrix (Table 2) resulting from the individual optimization of each objective.

376 Table 2.

377 Pay-off matrix for optimization of VRT process.

\begin{tabular}{l|ccc} 
& $\operatorname{Max}_{1}$ & $\operatorname{Max}_{2}$ & Min $_{3}$ \\
\hline$R_{C G}$ & 0.480 & 0.459 & 0.359 \\
$R_{Q S}$ & 0.354 & 0.385 & 0.038 \\
$t_{f}($ min $)$ & 157 & 200 & 91
\end{tabular}

378

379 This pay-off matrix corresponds to the Pareto-optimal extreme points, for which the 380 temperature profiles are shown in Figure 7. As expected, the minimum process time is 381 achieved with heating temperatures which reach the upper limit specified (for this 382 problem, $140 \stackrel{\circ}{\circ}$ ). Maximization of $R_{C G}$ and $R_{Q S}$ yield somewhat similar temperature 383 profiles, differing in the total process time. All the solutions belonging to the Pareto 384 front represent optimal alternatives whose temperature profiles are combinations of 385 those shown in Figure 7. The final operation policy will depend on the preferences of 386 the DM. 


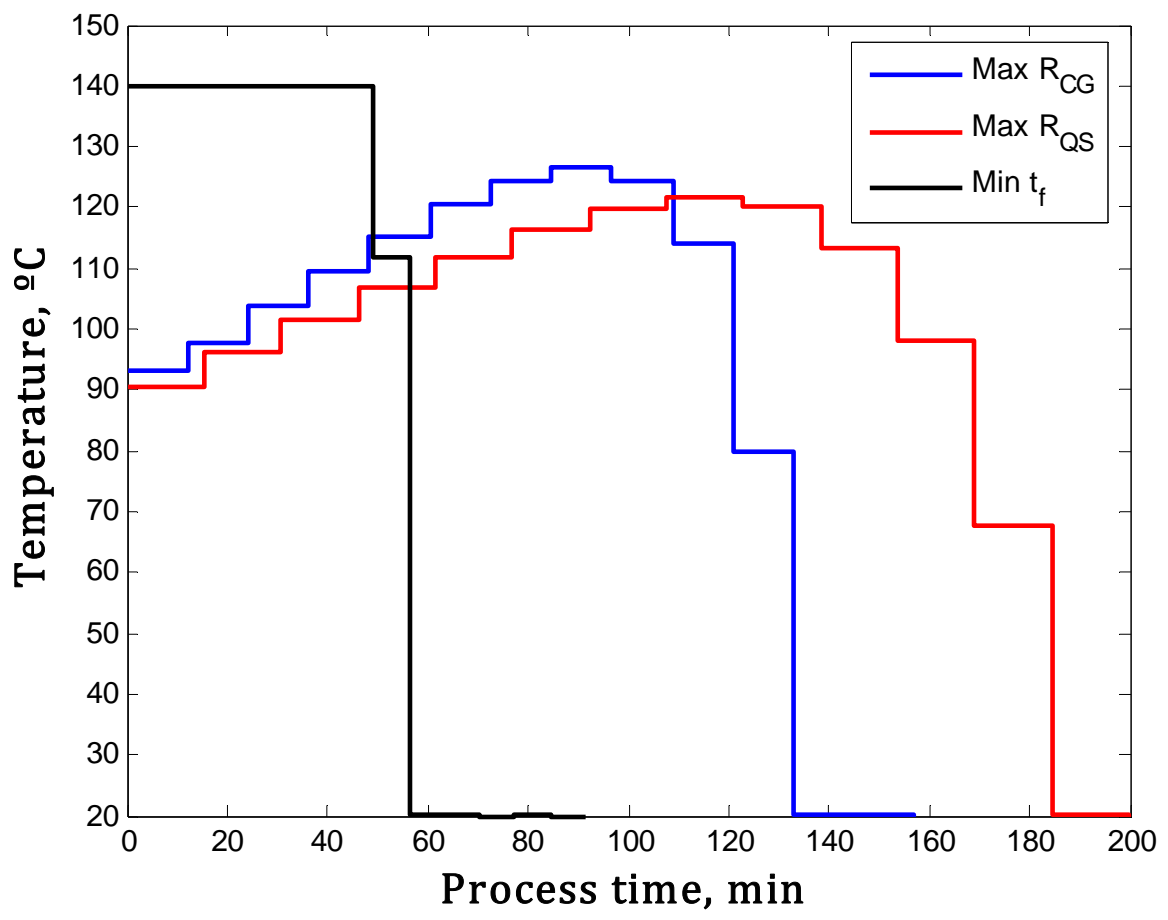

Figure 7. Temperature profiles for extreme points of the Pareto front.

389 Finally, we have compared the outcome of our approach with the results obtained 390 with the original NBI method and a well-known multi-objective genetic algorithm 391 (NSGA-II). For the original NBI, the algorithm DE was also used as the NLP solver, with 392 the same optimization settings as before. The equality constraints introduced by the 393 method were reformulated and handled very efficiently by means of a penalty 394 function (Sendín et al., 2006). Regarding the NSGA-II algorithm, and for the sake of 395 comparison, we applied a MATLAB implementation which was run for 5000 396 generations using a population size of 100 individuals.

397 As it can be observed, the solutions provided by the NSGA-II algorithm are not able to 398 capture the complete trade-off among the objectives, yielding also several dominated 399 points. On the other hand, results obtained with the NBI method are quite far from the 400 Pareto front, even when a GO solver is used. Thus, the approach described in this paper presents clear advantages over both NSGA-II and the original NBI formulation. 


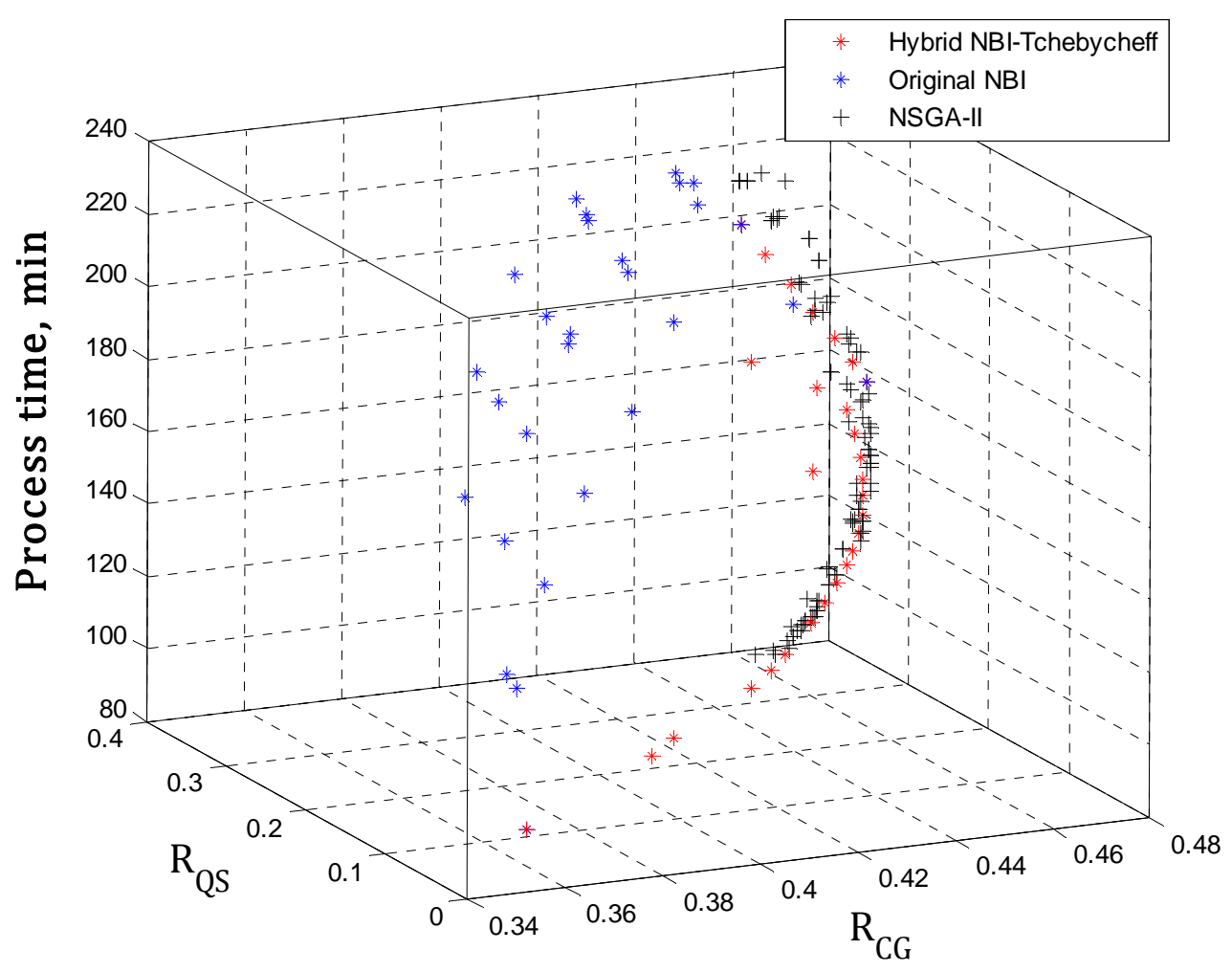

402

Figure 8. Comparison with the original NBI method and NSGA-II.

404 Regarding the performance of the NBI Weighted Tchebycheff approach in terms of 405 computational effort, convergence curves for three NLPs corresponding to different 406 regions of the Pareto front are depicted in Figure 9, which shows that the number of 407 function evaluations could be greatly reduced. It is worth noting that, since the NLPs 408 are solved sequentially, the initial populations include the optimal solutions of the sub409 problems which have been already solved. This is translated into a faster convergence 410 to the vicinity of the global minimum and an enhancement of the method efficiency. 


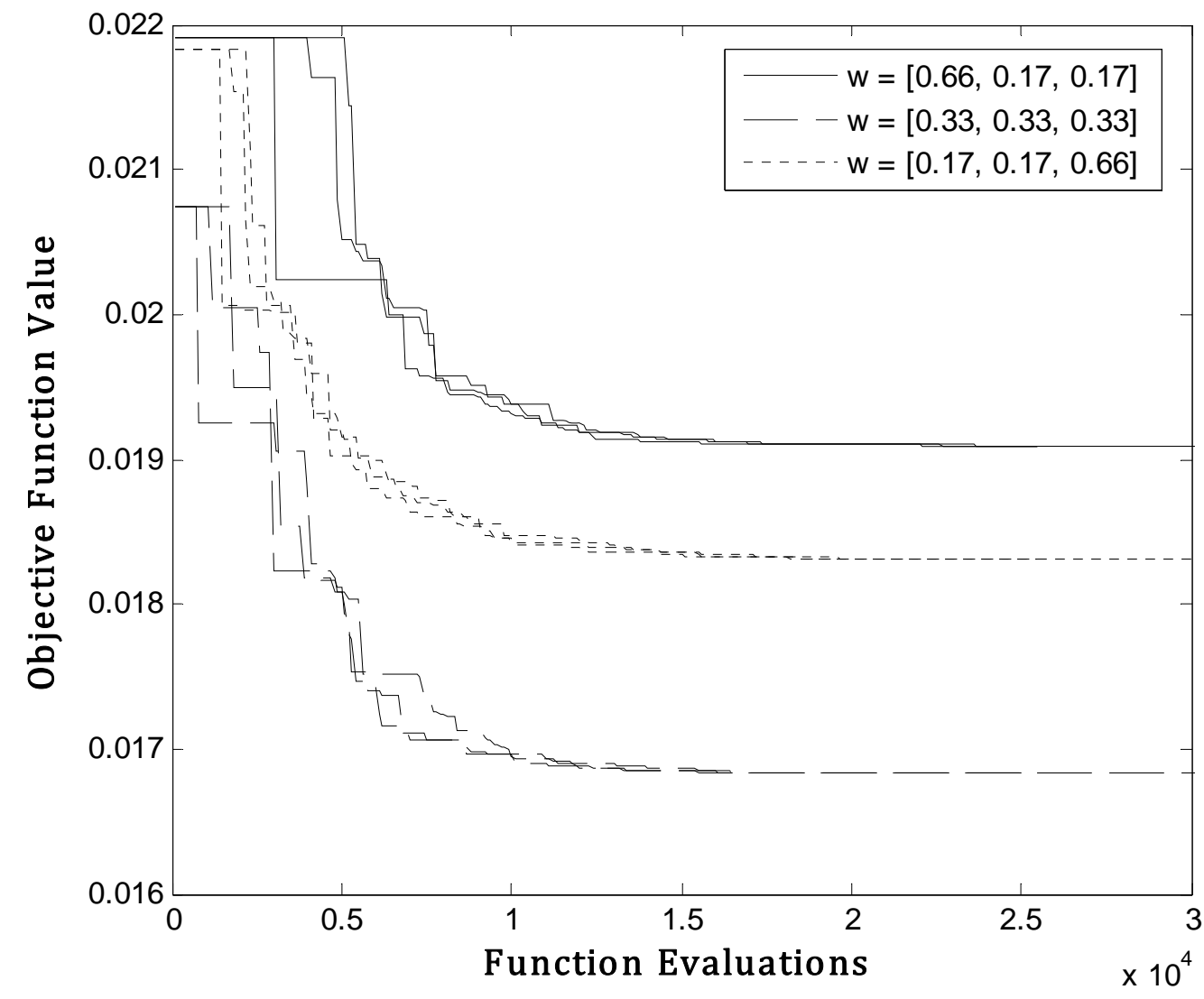

412

Figure 9. Convergence curves for selected NLPs derived from the NBI-Weighted

413

Tchebycheff approach.

414 


\section{CONCLUSIONS}

416 In this work, a novel multi-criteria optimization method was successfully applied to 417 the thermal processing of foods, where the simultaneous maximization of the 418 retention of several nutrients and quality factors and the minimization of total process 419 time was considered.

420 The new strategy, based on a combination of two well-known mathematical 421 programming techniques, has proved to be efficient and robust when applied to the 422 non-linear dynamic model considered. After generating the complete set of Pareto423 optimal solutions for several case studies, it can be readily used to choose a suitable 424 compromise between the objectives. Thus, this new technique can be used as the 425 computational engine of a powerful decision support system (DSS) for thermal 426 processing of foods.

427 Since the new technique can be coupled with single-objective, stochastic, GO 428 solvers, this method can adequately deal with more complex and large dynamic 429 systems involving partial differential equations. Furthermore, the new approach has 430 proved to be superior to the original NBI method, which fails to obtain global Pareto431 optimal solutions. It also presents clear advantages over other well-known methods, 432 like NSGA-II, when it comes to generate a fair good and easy-to-use representation of 433 the Pareto front capturing the complete trade-off among the objectives. 
Balsa-Canto, E., Alonso, A.A. \& Banga, J.R. (2002a). A novel, efficient and reliable method for thermal process design and optimization. Part 1: theory. Journal of Food Engineering, 52, 227-234.

Balsa-Canto, E., Alonso, A.A. \& Banga, J.R. (2002b). A novel, efficient and reliable method for thermal process design and optimization. Part 2: applications. Journal of Food Engineering, 52, 235-247.

Banga, J.R., Perez-Martin, R.I., Gallardo, J.M. \& Casares, J.J. (1991). Optimization of the termal processing of conduction-heated canned foods: study of several objective functions. Journal of Food Engineering, 14, 25-51.

Banga, J.R., Alonso, A.A., Gallardo, J.M. \& Martín, R.P. (1993). Kinetics of thermal degradation of thiamine and surface color in canned tuna. Z. Lebensm. Unters. Forsch., 197, 127-131.

Banga, J.R., Balsa-Canto, E., Moles, C.G. \& Alonso, A.A. (2003). Improving food processing using modern optimization methods. Trends in Food Science \& Technology, 14, 131-144.

Bowman, V.J. (1976). On the relationship of the Tchebycheff norm and the efficient frontier of multiple-criteria objectives. In: Thiriez, H. and Zionts, S. (eds). Multiple Criteria Decision Making Lecture Notes in Economics and Mathematical Systems, 130 (pp. 76-85). Springer-Verlag, Berlin.

Chalabi, Z.S., van Willigenburg, L.G. \& Van Straten, G. (1999). Robust optimal receding horizon control of the thermal sterilization of canned foods. Journal of Food Engineering, 40(3), 207-218.

Chen, C.R. \& Ramaswamy, H.S. (2002). Modeling and optimization of variable retort temperature (VRT) thermal processing using coupled neural networks and genetic algorithms. Journal of Food Engineering, 53, 209-220.

Coello, C.A.C. (1999). A comprehensive survey of evolutionary-based multiobjective optimization techniques. Knowledge \& Information Systems. An International Journal, 1, 269.

Das, I. \& Dennis, J.E. (1998). Normal-Boundary Intersection: A new method for generating the Pareto surface in nonlinear multicriteria optimization problems. SIAM J. Optimization, 8, 631-657.

Deb, K. (2001). Multi-objective optimization using evolutionary algorithms. Wiley, Chichester, England.

Durance, T.D. (1997). Improving canned food quality with variable retort temperature processes: a review. Trends in Food Science \& Technology, 8(4), 113-118.

Erdogdu,F. \& Balaban, M.O. (2003). Complex method for nonlinear constrained multicriteria (multi-objective function) optimization of thermal processing. Journal of Food Process Engineering, 26, 357-375. 
Fryer, P. \& Robbins, P. (2005). Heat transfer in food processing: ensuring product quality and safety. Applied Thermal Engineering, 25, 2499-2510.

García, M.-S. G., Balsa-Canto, E., Alonso, A.A. \& Banga, J.R. (2006). Computing optimal operating policies for the food industry. Journal of Food Engineering, 74, 13-23.

Kiranoudis, C.T. \& Markatos, N.C. (2000). Pareto design of conveyor-belt dryers. Journal of Food Engineering, 46, 145-155.

Koski, J. \& Silvennoinen, R. (1987). Norm methods and partial weighting in multicriterion optimization of structures. International Journal for Numerical Methods in Engineering, 24, 1101-1121.

Lim, Y.I., Floquet, P. \& Joulia, X. (2001). Efficient implementation of the Normal Boundary Intersection (NBI) method on multiobjective optimization problems. Ind. Eng. Chem. Res. 40, 648-655.

Messac, A. \& Mattson, C.A. (2004). Normal Constraint Method with guarantee of even representation of complete Pareto frontier. AIAA Journal, 42, 2101.

Miettinen, K.M. (1999). Nonlinear multiobjective optimization. Kluwer Academic Publishers, Boston.

Miri, T., Tsoukalas, A., Bakalis, S., Pistikopoulos, E.N., Rustem, B. \& Fryer, P.J. (2008). Global optimization of process conditions in batch thermal sterilization of food. Journal of Food Engineering, 87, 485-494.

Noronha, J., van Loey, A., Hendrickx, M. \& Tobback, P. (1996). Simultaneous optimization of surface quality during the sterilization of packed foods using constant and variable retort temperature profiles. Food. Eng. J., 30, 283-297.

Ohlsson, T. (1980). Optimal sterilization temperatures for sensory quality in cylindrical containers. J. Food Sci., 45, 1517-1521.

Olmos, A., Trelea, I.C., Courtois, F., Bonazzi, C. \& Trystram, G. (2002). Dynamic optimal control of batch rice drying process. Drying Technology, 20(7), 1313-1345.

Schiesser, W.E. (1991). The numerical method of lines. Academic Press, New York.

Sendín, J.-O.H., Alonso,A.A. \& Banga, J.R. (2004). Efficient multi-criteria optimization of thermal processing of foods. Proceedings of "International Conference on Engineering and Food 9" (ICEF 9), March 7-11, Montpellier, France.

Sendín, J.-O.H., Otero-Muras, I., Alonso,A.A. \& Banga, J.R. (2006). Improved optimization methods for the multiobjective design of bioprocesses. Ind. Eng. Chem. Res., 45, 8594-8603.

Shukla, P.K. (2007). On the Normal Boundary Intersection method for generation of efficient front. Proceeding of the $7^{\text {th }}$ international conference on Computational Science, Part I: ICCS 2007, pp. 310-317, Beijing, China.

Simpson, R., Abakarov, A. \& Teixeira, A. (2008). Variable retort temperature optimization using adaptive random search techniques. Food Control, 19, 10231032. 
513

514

515

516

517

518

519

520

521

522

523

524

Storn, R. \& Price, K. (1997). Differential Evolution - A simple and efficient heuristic for global optimization over continuous spaces. Journal of Global Optimization, 11(4), 341-359.

Teixeira, A.A., Zinsmeister, G.E. \& Zahradnik, J.W. (1975). Computer simulation of variable retort control and container geometry and possible means of improving thiamine retention in thermally processed foods. J. Food Sci., 40, 656-659.

Vassiliadis, V.S., Pantelides, C.C. \& Sargent, R.W.H. (1994a). Solution of a class of multistage dynamic optimization problems. 1. Problems without path constraints. Ind. Eng. Chem. Res. 33(9), 2111-2122.

Vassiliadis, V.S., Pantelides, C.C. \& Sargent, R.W.H. (1994b). Solution of a class of multistage dynamic optimization problems. 2. Problems with path constraints. Ind. Eng. Chem. Res. 33(9), 2123-2133. 